\title{
Analysis of Viscous Losses in the Chemical Interface Layer of Love Wave Sensors
}

\author{
Bernhard Jakoby, Senior Member, IEEE, and Michael J. Vellekoop
}

\begin{abstract}
Love waves have been introduced as highly effective devices for liquid-sensing applications. For chemical sensors, a microacoustic delay line featuring a multilayered waveguide supporting a generalized Love wave mode can be used in an oscillator setup. The top layer of the waveguide is a chemical interface, which selectively adsorbs certain target molecules in the adjacent liquid. The increase in mass density caused by adsorption can be detected as changes in the oscillation frequency. Commonly used interface materials show viscoelastic losses leading to an unwanted damping of the wave. To keep the signal-to-noise ratio high, the total insertion loss of the delay line should be kept as low as possible. Furthermore, it must not exceed a certain value to allow the electronic circuitry to sustain the oscillation. We analyzed the viscoelastic losses, which strongly depend on the frequency being used. By means of the proposed theoretical approach, the maximum thickness of the interface layer can be determined not to exceed the losses that can be handled by the driving electronics.
\end{abstract}

\section{INTRODUCTION}

L OVE WAVES are advantageous for liquid-sensing ap$\checkmark$ plications because of their shear polarization, which avoids coupling to compressional wave modes in adjacent liquids and the resulting strong damping of the wave. Moreover, Love waves are effectively guided close to the sensing surface, which yields a high sensitivity of the wave to surface mass loadings [1]-[3]. The waveguiding structure of the used microacoustic Love wave delay line consists of a guiding layer deposited on a (piezoelectric) substrate. To prevent electrical interaction of the sensed liquid with the wave and the transducers, an additional metal layer can be placed on top of the guiding layer [4]. Finally, a chemical interface layer is deposited as the top layer of the stratified structure. This layer is in contact with the liquid being sensed, and it selectively adsorbs chemical species present in the liquid. The resulting mass loading leads to a reduction in wave velocity, which can be detected using the delay line in an oscillator configuration (Fig. 1).

(Bio)chemical interface films often show serious drawbacks in terms of aging and instability. To overcome these disadvantages, we apply molecularly imprinted materials as sensing films. They essentially consist of polymeric materials, showing significantly higher chemical stability

Manuscript received February 19, 1999; accepted November 23, 1999. This work was supported by the Brite-Euram project BE-951745:MIMICS.

The authors are with Delft University of Technology, Electronic Instrumentation Lab./DIMES, Mekelweg 4, 2628 CD Delft, The Netherlands (e-mail: Bernhard.Jakoby@bosch.com). Present address of B. Jakoby: Robert Bosch GmbH, Sensor Technology Center K8/SPP5, Tübinger Strasse 123, D-72762 Reutlingen, Germany.

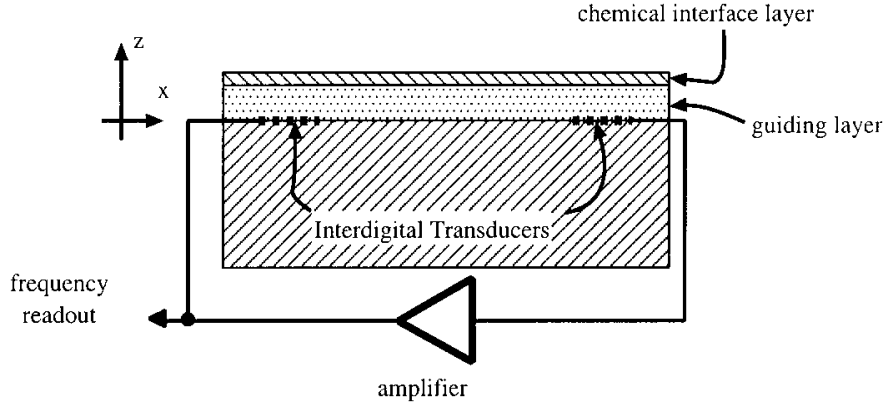

Fig. 1. Cross section of a Love wave delay line and schematic implementation in an oscillator.

and high specificity compared with other (bio)chemical interface materials. The process of molecular imprinting is based on the fabrication of a polymer, which comprises a certain amount of template molecules (i.e., the molecules to be detected). After polymerization, the template molecules can be washed out such that empty sites characteristic for the template molecule ("fingerprints") remain in the polymer. Upon re-exposure, template molecules are preferably absorbed into the empty sites imprinted in the polymer. In that manner, a selective film is realized on the basis of a polymer material. For more information on molecularly imprinted materials, see [5]. The physical properties of the imprinted polymer films resemble that of typical polymer coatings such as, for instance, polymethylmetacrylate (PMMA) films.

Because polymer films generally show viscoelastic behavior, the deposition of the film leads to a damping of the wave, which, together with the regular insertion loss of the device, has to be compensated by the amplifier in the oscillator circuit. In this contribution, we present analysis results on the magnitude of this damping, which, in turn, provide guidelines for the optimum design of the sensor device.

\section{Physical Impact of the Interface Layer}

The wave-guiding effect of the structure shown in Fig. 1 is based on the fact that the acoustic bulk shear wave velocity of the guiding layer material is lower than that of the substrate. As substrate material we used ST-cut quartz; the propagation direction was chosen orthogonal to the crystalline $\mathrm{X}$-axis. The bulk shear velocity in that direction equals about $5000 \mathrm{~m} / \mathrm{s}$. The guiding layer is made of $\mathrm{SiO}_{2}$, which has been deposited using Plasma Enhanced Chemical Vapor Deposition (PECVD). For PECVD $\mathrm{SiO}_{2}$, 
no reliable material parameters are available. As an approximation, the parameters for fused quartz can be used, which yield a bulk shear velocity of about $3800 \mathrm{~m} / \mathrm{s}$. The actually deposited PECVD films show even lower shear velocities [1].

In principle, the interface layer itself could also be used as a guiding layer (e.g., in [2], the application of a PMMA overlayer as guiding layer is discussed). However, as mentioned before, common interface materials (polymers) feature viscoelastic losses that would lead to a high damping of the wave. In [6], the damping induced by such a lossy overlayer is analyzed for the special case of an isotropic substrate. We decided to use both a guiding layer as well as an interface layer on top. To have the acoustic energy mainly concentrated in the guiding layer, the interface layer has to be kept thin compared with the guiding layer. Because polymers show very low bulk shear wave velocities (e.g., PMMA typically shows about $1100 \mathrm{~m} / \mathrm{s}$ ), a considerable amount of the guided energy can be concentrated in the interface layer. This effect increases with increasing frequency when the wave guiding effect generally becomes more pronounced.

\section{ANALYSIS}

To analyze the influence of viscoelastic losses in the chemical interface material on the wave, we calculate the field distribution in the waveguide. For an isotropic Love waveguide, this can be essentially done in closed form; only the propagation constant $\beta=2 \pi / \lambda,(\lambda=$ wavelength $)$ has to be obtained numerically from a dispersion equation. For the more general case of a piezoelectric (and hence anisotropic) substrate material, numerical methods have to be used. We used a spectral approach in which a harmonic time dependence $\exp (j \omega t)$ and a spatial dependence $\exp (-\gamma x)$ are assumed for all fields, ( $x$ denotes the propagation direction.) Introducing this into the field equations, the fields in the layer interfaces can be linked using propagator matrices [3], [7], [8]. For the space above the layers and the substrate, the fields are expanded into transverse modes showing evanescent behavior as $x$ approaches infinity. In that manner, a homogeneous linear system of equations can be obtained for a reduced set of field quantities. The solvability condition for this system yields the propagation constant $\gamma$. For a lossy waveguide, this implies the solution for the complex propagation constant $\gamma=\alpha+j \beta$, which, in general, yields a significant increase in computational effort compared with the lossless case where $\alpha=0$. Hence, we used a perturbation approach, in which, first, a lossless interface layer is assumed and then the specific losses in the interface layer are easily calculated by using the field solution the lossless guide as an approximation for the fields in the lossy guide. From the resulting loss per unit length in propagation direction, the damping constant $\alpha$ of the wave can be determined by [9]:

$$
\alpha=\frac{P_{d}}{2 P_{r}}
$$

Here, $P_{d}$ denotes the time-average power loss caused by viscous damping in the interface layer per unit of surface area in the interface plane ( $x y$ plane in Fig. 1) and $P_{r}$, is the power flow in $x$ direction in the lossless guide (per unit length in $y$ direction), which can be obtained by integrating the real part of the $\mathrm{x}$-component of the complex Poynting vector [10] over the waveguide cross section:

$$
P_{r}=\frac{1}{2} \operatorname{Re}\left\{j \omega \int\left(u_{2}^{*} T_{21}-\phi D_{1}^{*}\right) d z\right\} .
$$

Here, $T_{21}$ denotes the $y x$ component of the stress tensor $T_{i j}, u_{2}$ is the $y$ component of the displacement vector $u_{i}, \phi$ is the electric potential, and $D_{1}$ is the $x$ component of the electric displacement. The asterisk denotes the complex conjugate. In this expression, we considered that we are dealing with a pure $y$-polarized shear wave $\left(u_{1}=u_{3}=0\right)$. Eq. (2) shows that the electric fields traveling along with the wave, because of the piezoelectricity of the substrate, also lead to a contribution in the power flow even in the region above the guide (in the liquid) where no mechanical displacement is present ${ }^{1}$. Commonly, these electric fields in the liquid region are weak (especially for low coupling substrates such as quartz) such that their contribution to the power flow can be neglected and the integration can be restricted to the solid waveguide cross section. As mentioned before, it can also be advantageous to use an electric shielding layer on the guiding layer to prevent the electric interaction of the wave with the liquid [4]. In that case, the electric field in the liquid vanishes completely.

To characterize the losses in the interface material, we introduce a loss tangent $\tan \delta$ that characterizes the losses in the material. Phenomenologically, viscoelastic losses enter in the constitutive equation (Hooke's law) in frequency domain as follows [10]:

$$
T_{i j}=\sum_{k l}\left(c_{i j k l} S_{k l}+j \omega \eta_{i j k l} S_{k l}\right)
$$

where $S_{k l}$ denotes the strain tensor, $S_{k l}=\frac{1}{2}\left(\partial u_{k} / \partial x_{l}+\right.$ $\left.\partial u_{l} / \partial x_{k}\right) . c_{i j k l}$ is the elasticity tensor, and $\eta_{i j k l}$ represents the viscosity constants describing the viscoelastic losses in the medium. For isotropic media, the number of independent elastic constants can be reduced to two independent elastic constants, which are often taken to be the Lamé constants $\lambda$ and $\mu$ [10]. (The Lamé constant $\lambda$ must not be confused with the wavelength.) For pure shear movements, only $\mu$ is relevant. Thus, viscous losses can be considered by introducing a complex Lamé constant $\hat{\mu}=\mu+j \omega \eta_{\mu}$, where $\eta_{\mu}$ denotes the viscosity parameter associated with $\mu$. The power loss $P_{d}$, in (1) can then be obtained by integrating the time average power loss density [10] with

\footnotetext{
${ }^{1}$ In this analysis, we assume ideal, i.e., non-viscous, liquids (see subsequent remarks).
} 
respect to $z$ :

$$
P_{d}=\frac{\omega^{2} \eta_{\mu}}{2} \int_{\substack{\text { interface } \\ \text { layer }}}\left(\left|\frac{\partial u_{2}}{\partial z}\right|^{2}+\beta^{2}\left|u_{2}\right|^{2}\right) d z
$$

where $\beta$ is the unperturbed propagation constant obtained for the lossless waveguide. We define the loss tangent as the ratio of the imaginary to the real part of $\hat{\mu}$ :

$$
\tan \delta=\frac{\omega \eta_{\mu}}{\mu} .
$$

For purely elastic media, we hence have $\tan \delta=0$.

We note that, when immersing the delay line in liquid, another damping mechanism appears. Because of the viscosity of non-ideal liquids, a liquid layer gets entrained with the shear movement at the sensing surface, which leads to viscous losses in the liquid as well. These losses can also be analyzed using perturbation theory approaches [11], [12]. In the present analysis, we only want to consider the effects of viscous losses in the chemical layer such that we assume loading with an ideal, non-viscous liquid.

\section{Results}

To illustrate the typical behavior, we show some results for a Love wave waveguide with ST quartz substrate, $\mathrm{SiO}_{2}$ guiding layer (material parameters for fused quartz have been assumed), and a PMMA overlayer, representing the acoustic parameters of the interface layer. The thickness of the guiding layer was chosen as $5 \mu \mathrm{m}$ and that of the PMMA overlayer was $1 \mu \mathrm{m}$, which resembles typically used values.

The previously mentioned perturbation approach for the calculation yields a linear dependence of the attenuation $\alpha$ on $\tan \delta$. In reality, this is valid only for small $\tan \delta$ (in accordance with the perturbation principle). To obtain an idea about the validity range of the perturbation theory, we analyzed a corresponding approximate isotropic waveguide, allowing the application of a straightforward exact analysis approach, and compared the results to our perturbation approach. In this isotropic approximation, the ST quartz substrate is replaced by an isotropic medium with a bulk shear velocity of $5060 \mathrm{~m} / \mathrm{s}$ and a mass density of $\rho=2.65 \times 10^{3} \mathrm{~kg} / \mathrm{m}^{3}$ corresponding to the values in ST-cut quartz in the considered propagation direction. The analysis has been performed at $\omega=1.5 \times 10^{9} \mathrm{~s}^{-1}$ and $2 \times 10^{9} \mathrm{~s}^{-1}$. The results shown in Fig. 2 give the resulting damping $\alpha$, for increasing $\tan \delta$. The perturbation theory yields the proper result up to about $\tan \delta=0.1$, which corresponds to a fairly lossy material, and $\tan \delta=0.7$ for the lower and higher frequency, respectively.

Having verified the boundaries of the perturbation approach, we turn to the actual waveguide with piezoelectric substrate. To study the effect of energy concentration in the overlayer with increasing frequency, we calculated the power flux density in $x$ direction (i.e., the real part of the $x$ component of the electroacoustic Poynting vector,
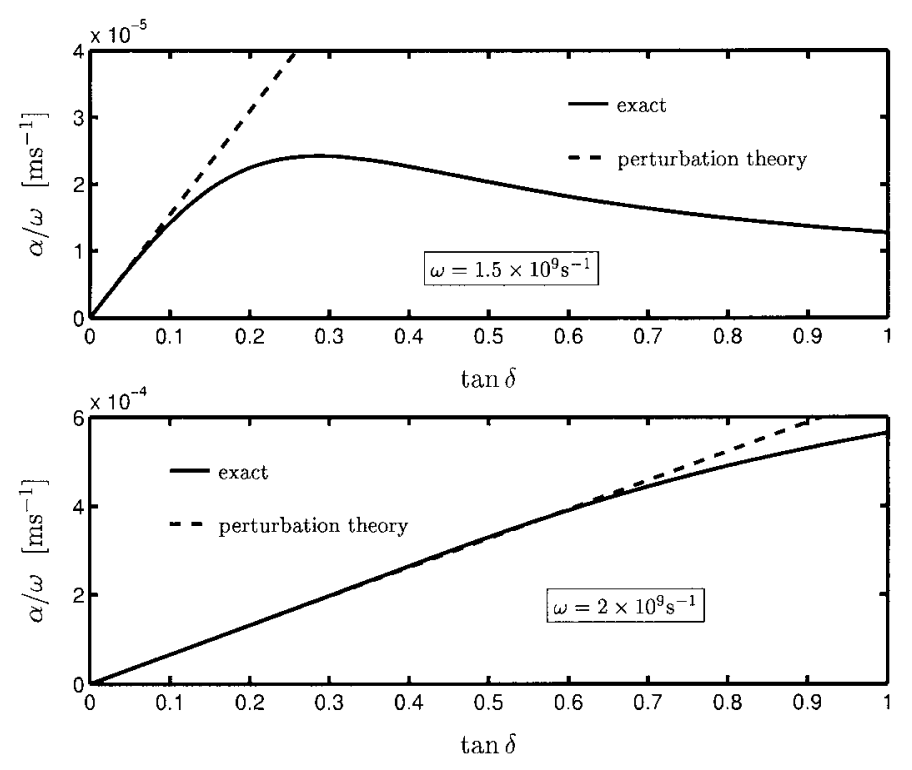

Fig. 2. Comparison of perturbation theory and exact approach for an approximate isotropic waveguide at two frequencies.

$\left.\operatorname{Re}\left\{P_{x}\right\}\right)[10]$. Integrating $\operatorname{Re}\left\{P_{x}\right\}$ with respect to $z$ over the substrate region $(z=-\infty \ldots 0)$ and the layer regions $(z=0 \ldots 5 \mu \mathrm{m}$ and $z=5 \ldots 6 \mu \mathrm{m}$ for the guiding layer and the PMMA layer, respectively) yields the associated partial fluxes in those regions. Fig. 3 shows the distribution of the total power flux to substrate and layers. The partial fluxes are scaled such that the total flux equals one. It can be seen that at the lowest frequencies shown, the flux is already fairly concentrated in the guiding layer. Increasing frequency yields a further increase of flux concentration in the guiding layer and a reduction in the substrate. At the same time, the flux in the PMMA overlayer increases and, from about $\omega=1.4 \times 10^{9} \mathrm{~s}^{-1}$, the flux in the guiding layer is decreasing. and more and more flux is taken over by the PMMA overlayer. Accordingly, we can see an increase in the damping constant $\alpha$ (shown in the subsequent figure). Note that the damping is given in a scaled manner $\alpha / \tan \delta$, where $\tan \delta$ is frequency dependent in general. For approximately constant elasticity and viscosity parameters, $\tan \delta$ would increase linearly with $\omega(4)$. Hence, the increase in $\alpha$ with frequency is actually stronger than that shown for the ratio $\alpha / \tan \delta$, in Fig. 3 .

Let us look at the displacement and flux distributions over the waveguide cross section at two particular frequencies. Fig. 4 and 5 show the distribution of the shear displacement amplitude and the power flux across the waveguide at $\omega=1.5 \times 10^{9} \mathrm{~s}^{-1}(f \approx 239 \mathrm{MHz})$ and $\omega=2 \times 10^{9} \mathrm{~s}^{-1}(f \approx 318 \mathrm{MHz})$. As can be seen in Fig. 3, the flux at the lower frequency is mainly concentrated in the guiding layer, whereas, at the higher frequency, it is essentially concentrated in the PMMA layer. But, even at the lower frequency, the displacement amplitude in the PMMA overlayer is already considerably higher compared with that in the guiding layer. But because of the smaller $\mu$ in PMMA, this does not necessarily cause a higher flux amplitude. At the considered frequency, the flux in the 

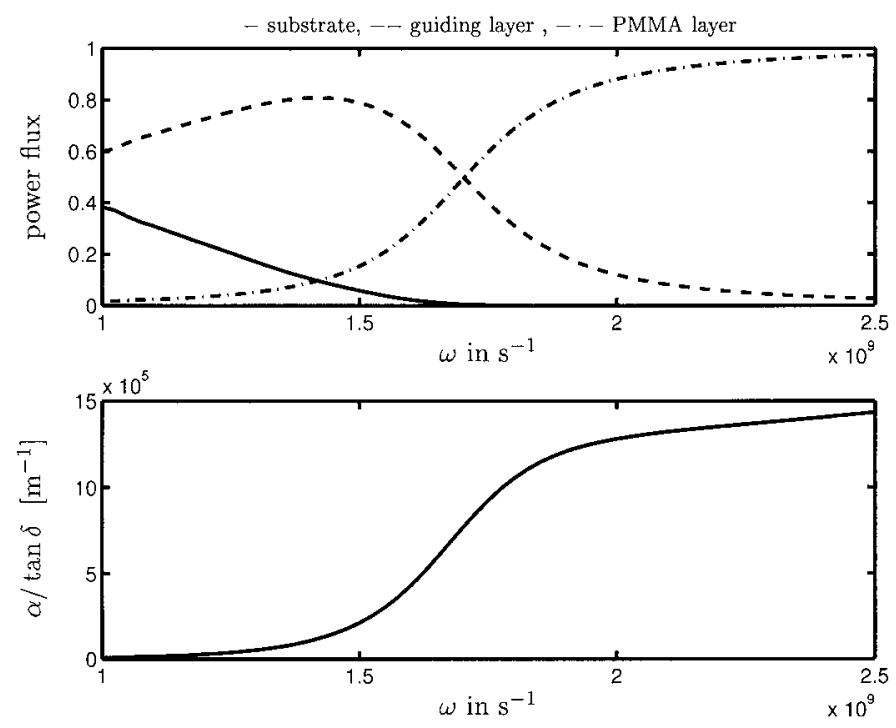

Fig. 3. Distribution of power flux in substrate and layers for varying frequency $\omega$ (upper figure); damping coefficient $\alpha$ in the same frequency range (lower figure).

overlayer is still smaller than that in the guiding layer. Note that this influence of the elastic constants on the power flux also yields a discontinuity of the flux at medium boundaries $(z=0$, and $z=5 \mu \mathrm{m})$; the displacement is continuous.

To illustrate the application of these results for the estimation of overlayer-induced damping, assume that the PMMA has $\eta_{\mu}=0.01 \mathrm{Ns} / \mathrm{m}^{2}$, a typical value for viscoelastic non-crystalline insulators [10]. With $\mu=1.43 \times$ $10^{9} \mathrm{~N} / \mathrm{m}^{2}$, we obtain from (4) that $\tan \delta=\omega \times 7 \times 10^{-12} \mathrm{~s}$. For instance, at $\omega=1.5 \times 10^{9} \mathrm{~s}^{-1}(f \approx 239 \mathrm{MHz})$, we would thus have $\tan \delta \approx 0.01$. In Fig. 3, we find $\alpha / \tan \delta \approx 2 \times 10^{5} \mathrm{~m}^{-1}$ at the considered frequency and, hence, $\alpha \approx 2000 \mathrm{~m}^{-1}$, which corresponds to a damping of $17300 \mathrm{~dB} / \mathrm{m}$. For a delay line with a length of $3 \mathrm{~mm}$, this would yield an additional device insertion loss of $52 \mathrm{~dB}$ because of viscoelastic damping. If we assume that the sensor electronics (amplifier in the oscillator configuration) can compensate for a maximum of $25 \mathrm{~dB}$ of additional insertion loss (typical value), then we find that the allowed maximum operation frequency is about $\omega=1.4 \times 10^{9} \mathrm{~s}^{-1}$ $(f \approx 223 \mathrm{MHz})$. For common acoustic devices, the damping induced by viscoelastic losses increases with about the square of the frequency [10]; in our case, this increase is even stronger in the utilized frequency range because of the previously described changes in flux distribution with increasing frequency. Hence, care has to be taken in the selection of the operation frequency of the device as illustrated by the previous example.

Finally, we note that a critical point in the accuracy of the analysis is the knowledge of material data (especially viscoelastic parameters such as $\eta_{\mu}$ ) for thin films. Such material data are not yet available such that typical values and expected ranges of values (e.g., for the loss tangent) had to be used in the previous analysis. To support the application of the presented analysis approach further, ac-

\section{Displacement distribution at two different frequencies}
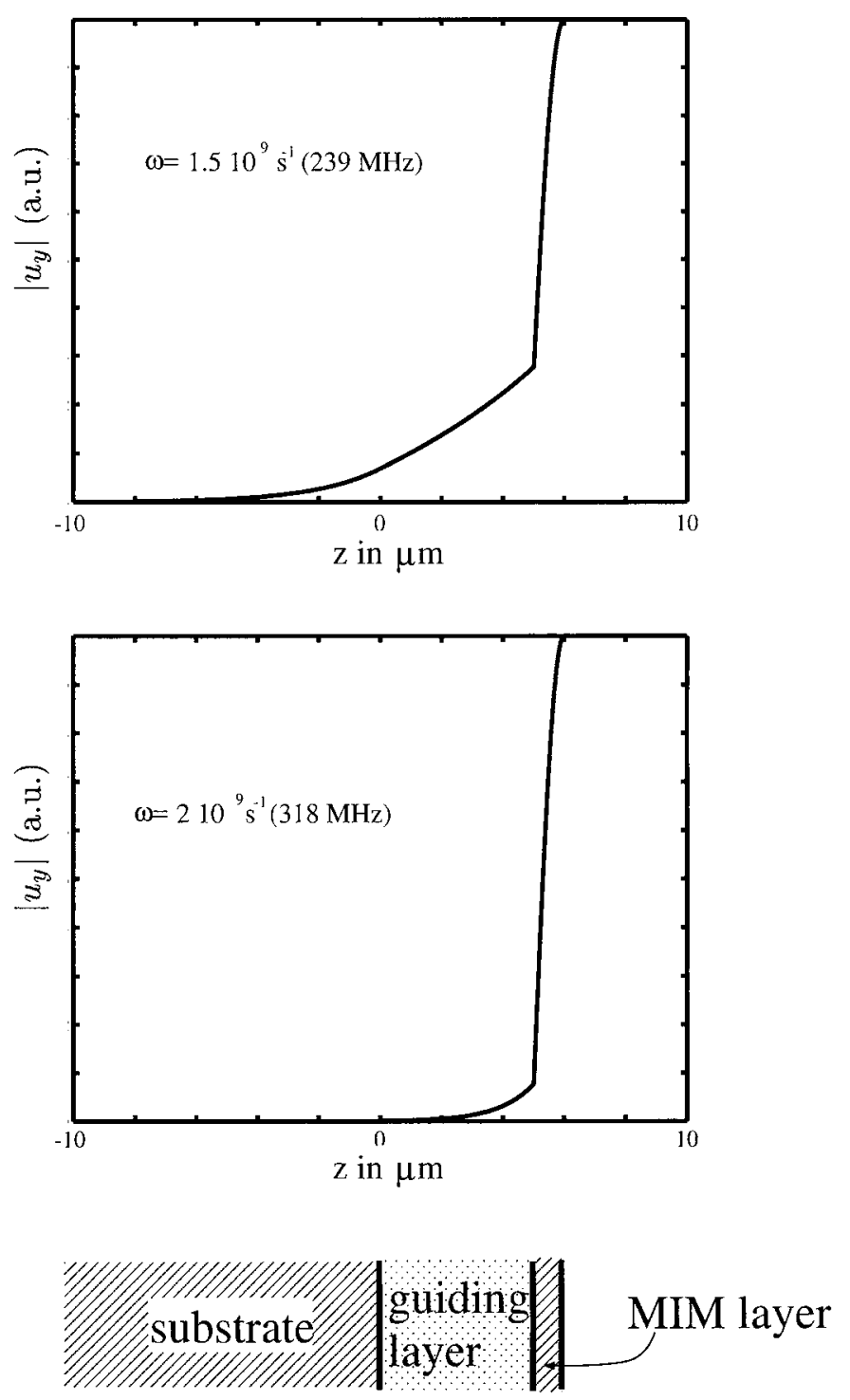

Fig. 4. Distribution of the shear displacement amplitude $u_{2}$ over the depth $z$ of the waveguide at $\omega=1.5 \times 10^{9} \mathrm{~s}^{-1}$ (upper figure) and $\omega=2 \times 10^{9} \mathrm{~s}^{-1}$. The substrate/guiding layer and the guiding layer/interface layer boundaries are located at $z=0$ and $z=5 \mu \mathrm{m}$, respectively.

tivities in the area of high frequency viscoelastic thin film characterization are thus highly desirable.

\section{Summary And Conclusions}

We discussed the physical influence of a chemically selective interface layer used on a microacoustic Love wave chemical sensor. Generally, an increase of the operation frequency can substantially increase the sensitivity of the sensor as the acoustic energy gets concentrated close to the sensing surface. On the other hand, viscous losses in the organic chemical interface layer lead to a strong increase of the damping for increasing frequency. The latter yields an increased device insertion loss that has to be compensated 


\section{Power flux distribution at two different frequencies}
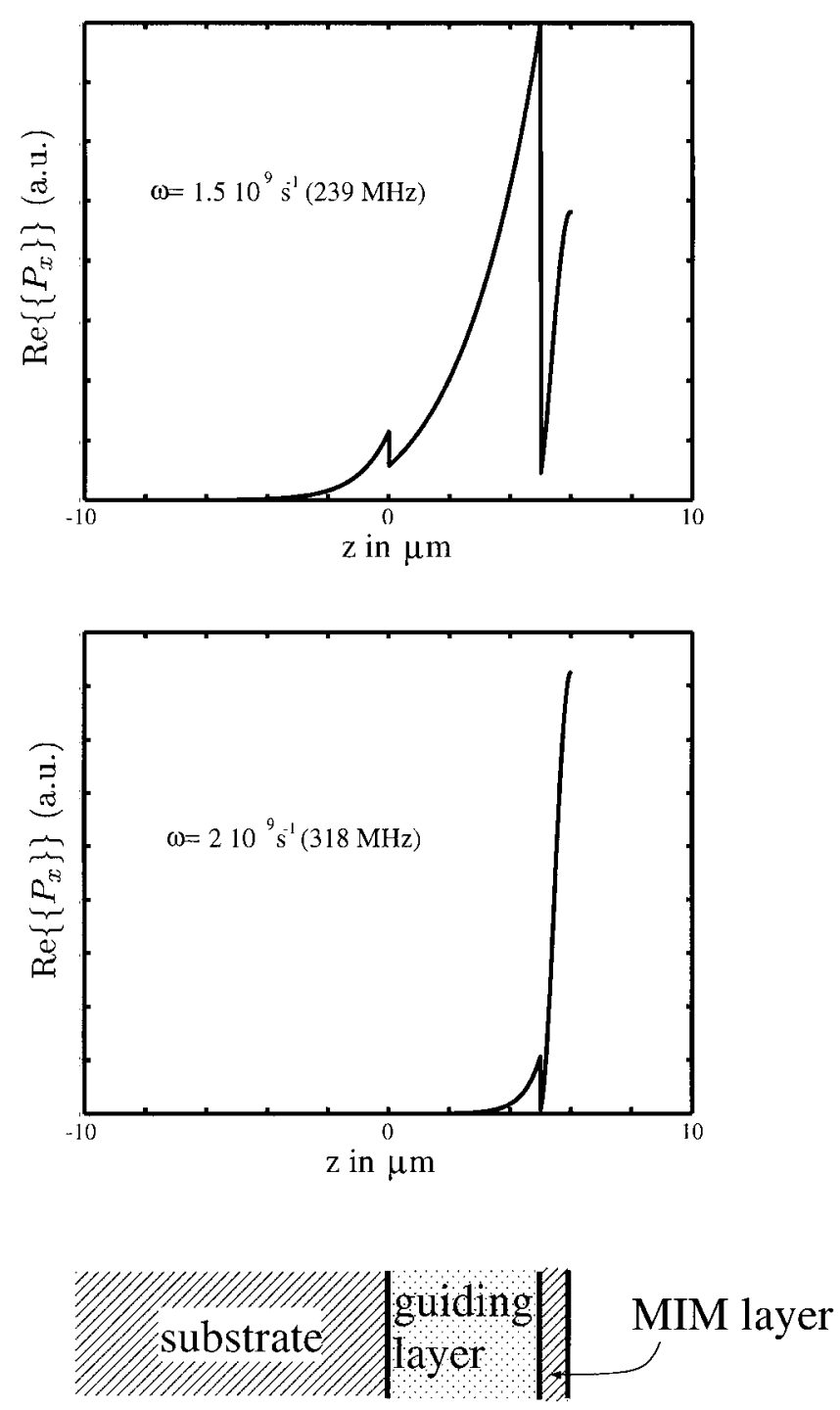

Fig. 5. Distribution of power flux (real part of Poynting vector $x$ component) over the depth $z$ of the waveguide at $\omega=1.5 \times 10^{9} \mathrm{~s}^{-1}$ (upper figure) and $\omega=2 \times 10^{9} \mathrm{~s}^{-1}$.

by the sensor electronics. The frequency-dependent acoustic energy distribution in the device has been analyzed, and the resulting viscoelastic damping has been determined using a perturbation theory approach. We showed that, using this approach, the maximum thickness of the chemical interface layer can be determined in order not to exceed the allowed maximum insertion loss permitted by the driving electronics.

\section{REFERENCES}

[1] G. Kovacs, G. W. Lubking, M. J. Vellekoop, and A. Venema, "Love waves for (bio)chemical sensing in liquids," in Proc. IEEE Ultrason. Symp., Tucson, AZ, pp. 281-285, 1992.

[2] E. Gizeli, A. C. Stevenson, N. J. Goddard, and C. R. Lowe, "A novel Love-plate acoustic sensor utilising polymer overlays," IEEE Trans. Ultrason., Ferroelect., Freq. Contr., vol. 39, no. 5, pp. 657-659, 1992.
[3] B. Jakoby and M. J. Vellekoop, "Properties of Love waves: Applications in sensors," Smart Mater. Struct., vol. 6, pp. 668-679, 1997.

[4] "Analysis and optimization of Love wave liquid sensors," IEEE Trans. Ultrason., Ferroelect., Freq. Contr., vol. 45, pp. 12931302, Sept. 1998.

[5] K. Mosbach and O. Ramstrom, "The emerging techniques of molecular imprinting and its future impact on biotechnology," Biotechnoloy, vol. 14, no. 2, pp. 163-170, 1996.

[6] P. Kielczyński and J.D.N. Cheeke, "Love waves propagation in viscoelastic media," in Proc. IEEE Ultrason. Symp., Toronto, Canada, pp. 437-440, 1997.

[7] A. H. Fahmy and E. L. Adler, "Propagation of acoustic surface waves in multilayers: A matrix description," Appl. Phys. Lett., vol. 22, pp. 495-497, May 1973.

[8] E. Langer, "Fundamental analysis of surface acoustic wave propagation," Archiv für Elecktronik und Übertragungstechnik, vol. 44, no. 3, pp. 225-232, 1990.

[9] B. A. Auld, Acoustic Fields in Solids, vol. II, New York: John Wiley \& Sons, 1973.

[10] — Acoustic Fields in Solids, vol. I, New York: John Wiley \& Sons, 1973.

[11] S. J. Martin, A. J. Ricco, T. M. Niemczyk, and G. C. Frye, "Characterization of SH acoustic plate mode liquid sensors," Sens. Actuators, vol. 20, pp. 253-268, 1989.

[12] B. Jakoby and M. J. Vellekoop, "Viscosity sensing using a Love wave device," Sens. Actuators A, vol. 68, pp. 275-281, 1998.

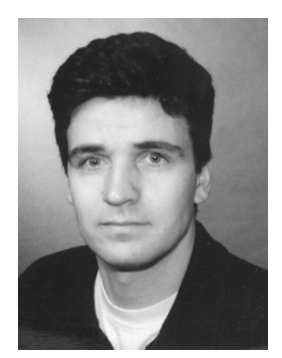

Bernhard Jakoby (S'91-A'95-SM'98) was born in Neuss, Germany, in 1966. He obtained the Dipl.-Ing. (M.Sc.) degree in Communication Engineering and the Ph.D. degree in Electrical Engineering from the Vienna University of Technology, Austria, in 1991 and 1994, respectively.

From 1991 to 1994, he worked as Research Assistant at the Institute of General Electrical Engineering and Electronics at the Vienna University of Technology, Austria. Later he obtained an Erwin Schrödinger grant from the Austrian Fund for Scientific Research (FWF) to perform research on the electrodynamics of complex media at the Department of Information Technology at the University of Ghent, Belgium. From 1996 to 1999, he held the position of Research Associate and later Assistant Professor at the Electronic Instrumentation Laboratory of the Delft University of Technology, The Netherlands, working on the development of microacoustic sensors. Since, 1999, he has been with the Robert Bosch GmbH, Reutlingen, Germany, performing research and development on sensors for automotive applications.

Bernhard Jakoby is a Senior Member of the IEEE. His research interests are focused on theory and applications in the fields of electromagnetics, microacoustics, and integrated sensors.

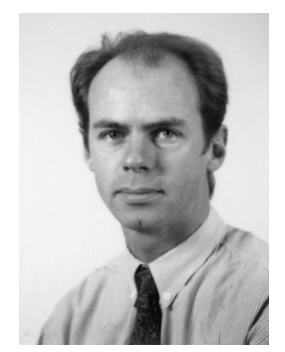

Micheal J. Vellekoop was born in Amsterdam in 1960. He received the BSc degree in Physics from the HTS Dordrecht, The Netherlands, and the $\mathrm{PhD}$ degree in Electrical Engineering from the Delft University of Technology, The Netherlands, in 1982 and 1994, respectively.

From 1982 to 1984 , he stayed with the Royal Netherlands Naval College as a reserve officer after which he joined the Electronic Instrumentation Laboratory at the Delft University of Technology to work in the field of acoustic wave sensors. In addition, from 1988 to 1996, he was Managing Director of Xensor Integration B. V., being involved in the development and production of silicon sensors and actuators. Currently, he leads the Physical Chemosensors and Microacoustic Devices Group of the Electronic Instrumentation Laboratory, DIMES, where he holds the position of Associate Professor. His recent research activities have been in the areas of microacoustic sensor systems for gas and liquid sensing applications, solid state sensor technology, and physical chemosensors. 